\title{
Donor Sertoli cells transplanted into irradiated rat testes stimulate partial recovery of endogenous spermatogenesis
}

\author{
Zhen Zhang ${ }^{1,2}$, Shan Shao ${ }^{1}$, Gunapala Shetty ${ }^{1}$ and Marvin L Meistrich ${ }^{1}$ \\ ${ }^{1}$ Department of Experimental Radiation Oncology, The University of Texas M. D. Anderson Cancer Center, Houston, \\ Texas 77030, USA and ${ }^{2}$ Monash Institute of Medical Research Center for Reproduction and Development, 27-31 \\ Wright Street, Clayton, Victoria 3168, Australia
}

Correspondence should be addressed to Z Zhang; Email: zhang8@hotmail.com

\begin{abstract}
Irradiation of rat testes leads to the failure to support differentiation of the surviving spermatogonia due to damage of the somatic environment. To determine the involvement of Sertoli cells in this somatic damage, we transplanted seminiferous tubule cells from normal immature GFP-transgenic rats into the testes of irradiated rats. The donor Sertoli cells colonized and developed in the host testes. In many seminiferous tubules, the donor Sertoli cells formed abnormal spherical structures in the lumen, but in some tubules they formed a normal-appearing epithelium, but with only isolated spermatogonia, on the basement membrane. When the donor cells were injected into the interstitial region of the testis, they formed tubule-like structures containing Sertoli cells and occasional isolated spermatogonia, both of donor origin. Surprisingly, in host tubules adjacent to these newly formed donor-cell tubules or adjacent to the endogenous tubules with abnormal donor Sertoli-cell structures, endogenous spermatogonia differentiated to the spermatocyte or even to spermatid stages. Around these newly donor cell-formed tubules and the host tubules with abnormal donor Sertoli-cell structures, many cells including macrophages, which perhaps represented chronic inflammation, accumulated in the interstitium. We conclude that the donor Sertoli cells that colonized the seminiferous tubules did not directly support recovery of spermatogenesis. Instead, the colonizing Sertoli cells acted indirectly on the interstitium to stimulate localized differentiation of endogenous spermatogonia.
\end{abstract}

Reproduction (2009) 137 497-508

\section{Introduction}

Spermatogenesis occurs in the enclosed environment of the seminiferous tubule and directly depends on the close and supporting relationship between the spermatogenic and Sertoli cells and on paracrine or indirect actions of other testicular somatic cells. Dysfunction of the somatic environment could lead to failure of normal spermatogenesis to proceed. For example, in irradiated rat testes, the surviving spermatogonial stem cells are capable of self-renewal and proliferation, but they are unable to differentiate and instead undergo apoptosis (Kangasniemi et al. 1996, Meistrich \& Shetty 2003). In an attempt to determine whether the radiation damaged the spermatogonia or the somatic environment, we recently transplanted spermatogonia from immature or irradiated adult green fluorescent protein (GFP)-transgenic rats into irradiated $\mathrm{LBNF}_{1}$ rats or nude mice respectively. We found that the irradiated spermatogonial stem cells could colonize and differentiate in the permissive murine host, but the irradiated rat testes did not support the differentiation of normal, functional, transplanted spermatogonial stem cells (Zhang et al. 2007). Although we showed that the defect in the somatic environment could be reversed by suppressing testosterone levels, the cell type and molecular factors responsible for the defective spermatogenic environment are not known.

To investigate the role of the Sertoli cell in spermatogenic development in the damaged testes, we took advantage of the ability of transplanted, immature Sertoli cells to develop in host testes. Previously it was shown that normal perinatal mouse Sertoli cells, transplanted into a testis with genetic defect in its own Sertoli cells, develop in the host testis and support complete spermatogenesis in a few tubules, which was sufficient to obtain enough testicular sperm for successful fertilization by intracytoplasmic sperm injection (Kanatsu-Shinohara et al. 2005). The percentage of tubules populated by donor Sertoli cells was increased when the recipient was treated to deplete endogenous Sertoli cells (Shinohara et al. 2003). In addition to the Sertoli cells, donor myoid and fibroblast-like cells could also colonize the host's seminiferous tubules and divide them into 'minitubules', some of which contained early and occasionally differentiated spermatogonial cells (Shinohara et al. 2003). We also demonstrated that donor Sertoli cells from immature rats colonized within 
the seminiferous tubules of irradiated rats, but in that study we did not focus on the effect of this colonization on spermatogonial differentiation (Zhang et al. 2007).

In the present study, we further investigated the results of transplantation of Sertoli cells from immature normal rat testes into the seminiferous tubules or the interstitial compartment of irradiated rat testes to investigate whether or not donor Sertoli cells might repair the damaged spermatogenic environment in irradiated rat testis. We found donor Sertoli cells, in addition to colonization, development, and formation of colonies along the basement membrane and in the lumen of the seminiferous tubule, also formed de novo tubule-like structures in the interstitium. Endogenous spermatogenesis in the irradiated rat testes partially recovered 3 months after the transplantation and was in association with donor Sertoli cell colonization in non-physiological locations.

\section{Results}

\section{Donor cells within seminiferous tubules}

When donor seminiferous tubular cells from immature GFP-transgenic rats were injected into the seminiferous tubules of irradiated rat testes, most of the donor cells observed 13 weeks later were GFP-positive Sertoli cells (Fig. 1A, B, E, F, I, J, M, N and O). In all cases, the cells were identified as donor Sertoli cells by GFP positivity (Fig. 1A, E, I, M and O) and strong WT1 nuclear staining (Fig. 1B, F, J, N and O). Single or short chains of donor spermatogonia were occasionally found in the recipient's seminiferous tubules, as reported previously (Zhang et al. 2007), but these never produced any differentiated cells. None of these Sertoli cells appeared to be proliferating as assessed by proliferating cell nuclear antigen (PCNA) staining at the time of tissue harvest, 13 weeks after transplantation. By contrast, endogenous germ cells were actively proliferating (Fig. 1P).

The donor Sertoli cells showed three major patterns of colonization in the recipient's seminiferous tubules: clustered in the lumen of the seminiferous tubules (Fig. 1E-H, L, O and P), a layer of cells along basement membrane (Fig. 1I-K, $\mathrm{M}$ and $\mathrm{N}$ ), and a single layer or clusters of cells along original and newly formed basement membranes in segregated tubules (Fig. 1A-D).

The most frequent of these patterns consisted of Sertoli cells in the center of the tubule, forming spherical structures around a central core, which contained collagen-rich extracellular matrix (Fig. 1L), usually surrounded by a layer of actin-positive, presumably peritubular myoid cells of donor origin (Fig. 1H). Although it appears in many cross sections that the Sertoli cell colonies were in the lumens of tubules, the structure on which they formed was contiguous, in some sections, with the periphery of the tubule (not shown) and, in some planes of section, with the endogenous peritubular myoid cells (Fig. 1D).

Other tubules contained donor Sertoli cells that covered part or all of the original basement membrane of the tubule (Fig. 1I-K, M and N). The morphology of these Sertoli cells and the epithelium was very similar to that of the endogenous Sertoli cell epithelium of the irradiated rat except that many of the tubules that contained a high proportion of donor cells along the basement membrane had a higher density of Sertoli cells (Fig. $1 \mathrm{M}$ and $\mathrm{N}$ ) than did other atrophic tubules from the irradiated rat. In addition, these tubules occasionally contained spermatogonia (Fig. 1K, arrows).

Some tubules were essentially filled with donor Sertoli cells with their nuclei arranged along the basement membrane (Fig. 1A and B). In many cases, these tubules were invaded by peritubular (Fig. 1D) and fibroblasticlike cells that could segregate the tubule into 'minitubules' (Shinohara et al. 2003).

Because of the age range of the donors, we analyzed the cellular composition of smears of suspensions prepared from donor rats at different ages using antiWT1 and anti-smooth muscle $\alpha$-actin antibodies to identify Sertoli and myoid cells respectively. The percentage of Sertoli and myoid cells respectively were 66 and $21 \%$ in 5-day-old donors, 71 and $14 \%$ in 10-day-old donors, 68 and $10 \%$ in age 15 -day-old donors, and 44 and $7 \%$ in 21-day-old donors. Despite a more marked drop in myoid cells than of Sertoli cells with age, all patterns of colonization were observed in similar frequencies with the donor cells from different ages of rats.

\section{Donor cells in the interstitial compartment}

Irradiated rat testes, in which donor seminiferous tubular cells from immature GFP-transgenic rats were injected into the interstitium, either accidentally or deliberately, contained numerous irregular tubular-like structures 13 weeks after injection (Fig. 2). These structures contained primarily donor Sertoli cells, as evidenced by staining with anti-GFP (Fig. 2A, E, I and J) and anti-WT1 (Fig. 2B, $\mathrm{F}$ and $\mathrm{I}-\mathrm{L}$ ). At their periphery, these tubule-like structures always contained a layer of peritubular myoid cells, which sometimes was interrupted by a gap (Fig. 2D and $\mathrm{H}$, thick arrows). Most of the myoid cells in this layer were GFP negative (Fig. 2A and E) and were embedded in a collagen-rich basement membrane as indicated by trichrome staining (not shown). The Sertoli cells formed a layer near the basement membrane of the newly formed tubule structures (Fig. 2B and F).

When the cells were deliberately injected into the interstitial area, large areas of newly formed tubular structures, like those created by inadvertent interstitial injection, were formed surrounding the preexisting tubules (Fig. 2E-H). This difference in area was likely a result of the larger volume of donor cells injected into the interstitial compartment during deliberate injection. 

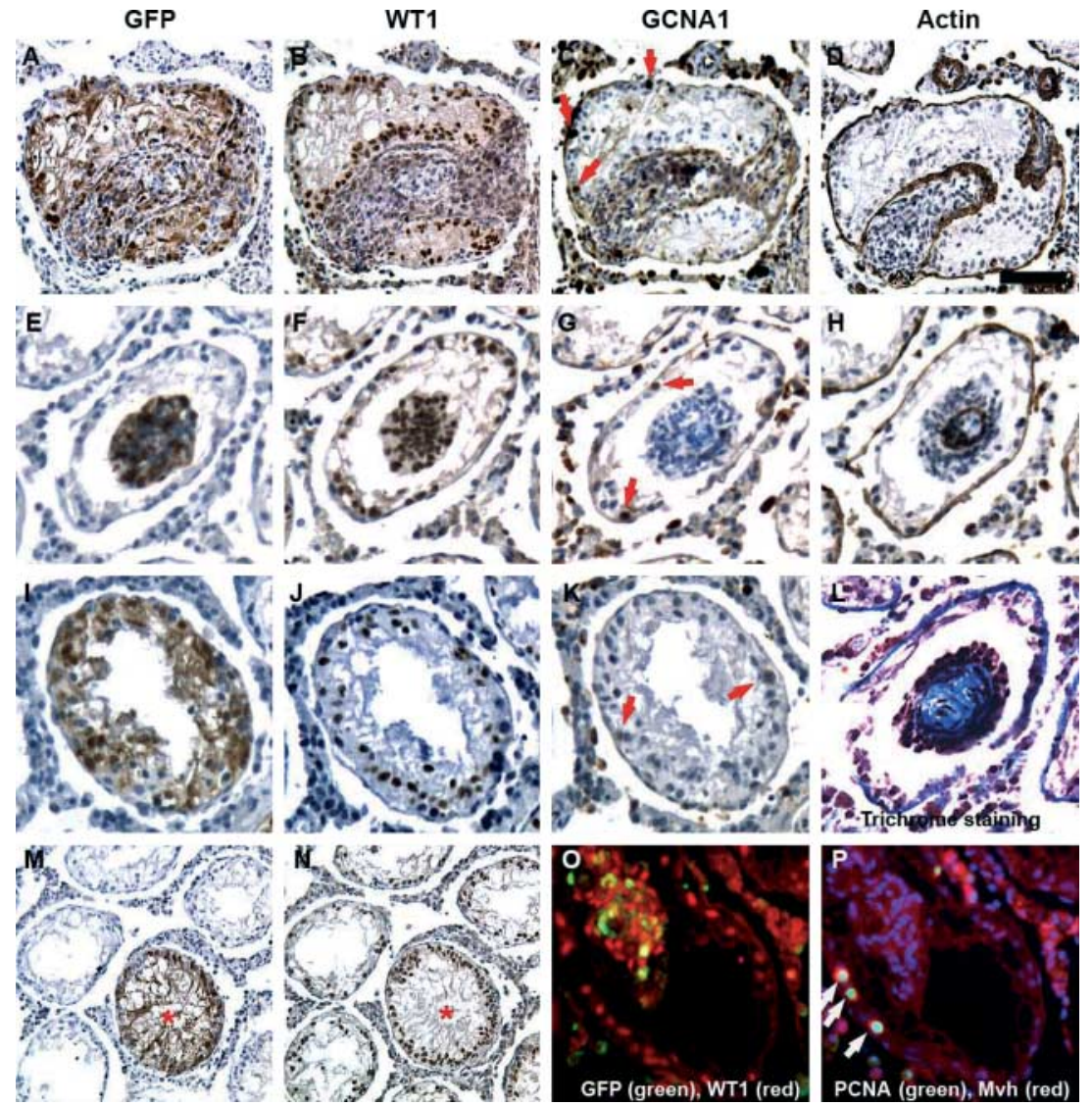

These newly formed tubules contained one layer of donor Sertoli cells, which were often densely packed against the newly formed basement membrane and less densely packed peripheral to the preexisting tubule (Fig. $2 \mathrm{l}$ and J). None of the Sertoli cells in the newly formed tubules were still proliferating, as assessed with PCNA staining at 13 weeks after transplantation (Fig. 2K and L). Germ cells, most likely of donor origin since endogenous spermatogonia are confined to the host tubules, were occasionally observed in these newly formed tubular structures, as indicated by nuclear GCNA1 staining that appeared stronger than the background staining (Fig. 2C and G, thin arrows).

\section{Increased host cellular material developing in the interstitium}

When cells were injected through the rete testis into the seminiferous tubules, there was an increase in cellularity around some of the tubules that contained colonies of donor Sertoli cells (Fig. 3A). When the cells were inadvertently injected interstitially through the rete testis area, the newly formed tubules were also often surrounded by an excess of interstitial cells (Fig. 3B). In both cases, these cells appeared to be produced as part of a chronic inflammatory reaction. Acute inflammation,
Figure 1 Characterization of various structures produced by Sertoli cells injected into seminiferous tubules by immunohistochemical staining. Donor cells were stained with GFP (A, E, I, M and O), Sertoli cells with WT1 (B, F, J, N and O), germ cells with GCNA1 (C, G and K) or Mvh (P), peritubular myoid cells with smooth muscle $\alpha$-actin ( $\mathrm{D}$ and $\mathrm{H})$, proliferating cells with antiPCNA (N), and collagen (blue) with trichrome stain (L). The following groups of panels are serial sections $(\mathrm{A}-\mathrm{D}),(\mathrm{E}-\mathrm{H}$ and $\mathrm{L}),(\mathrm{I}-\mathrm{K}),(\mathrm{M}$ and $\mathrm{N})$, and $(\mathrm{O}$ and $\mathrm{P})$. (A-D) The invagination of a tubule containing donor Sertoli cells and division into minitubules. (E-H, O and P) The structures formed by donor Sertoli cells in the lumen of tubules. (I-K, $M$ and N) An epithelium formed by donor Sertoli cells replacing most of the host Sertoli cells in these tubules (labelled with asterisks in $\mathrm{M}$ and $\mathrm{N}$ ). Arrows indicate spermatogonia. Bar $=100 \mu \mathrm{m}$ in all panels except $M$ and $N$, in which it represents $200 \mu \mathrm{m}$. characterized by very small, closely packed cells, was rarely seen with either intratubular or interstitial injection. Occasionally, inflammatory-like cells appeared to be invading and destroying the tubular structure (Fig. 3A, arrow). These reactions did not appear to be related to immunoincompatibility since the interstitial thickening was independent of the backcross generation of the rats from which the transplanted cells were obtained.

The newly formed interstitial cells were actively proliferating, as indicated by their positive staining for PCNA (Fig. 3D). Many of the cells appeared to originate from circulating monocytes or macrophages as demonstrated by positive ED1 staining (Fig. 3E). A smaller number of the interstitial cells were tissue-resident macrophages marked with the ED2 antibody (Fig. 3F).

\section{Stimulation of endogenous spermatogenesis: histo- logical observations}

When Sertoli cells were injected into the seminiferous tubules and colonized the lumen of the host tubules (Fig. 4A-C arrowheads), we were surprised to see differentiated germ cells (GFP negative and GCNA1 positive), which were present primarily in adjacent tubules in the cross section (Fig. 4A-C, asterisks), and 

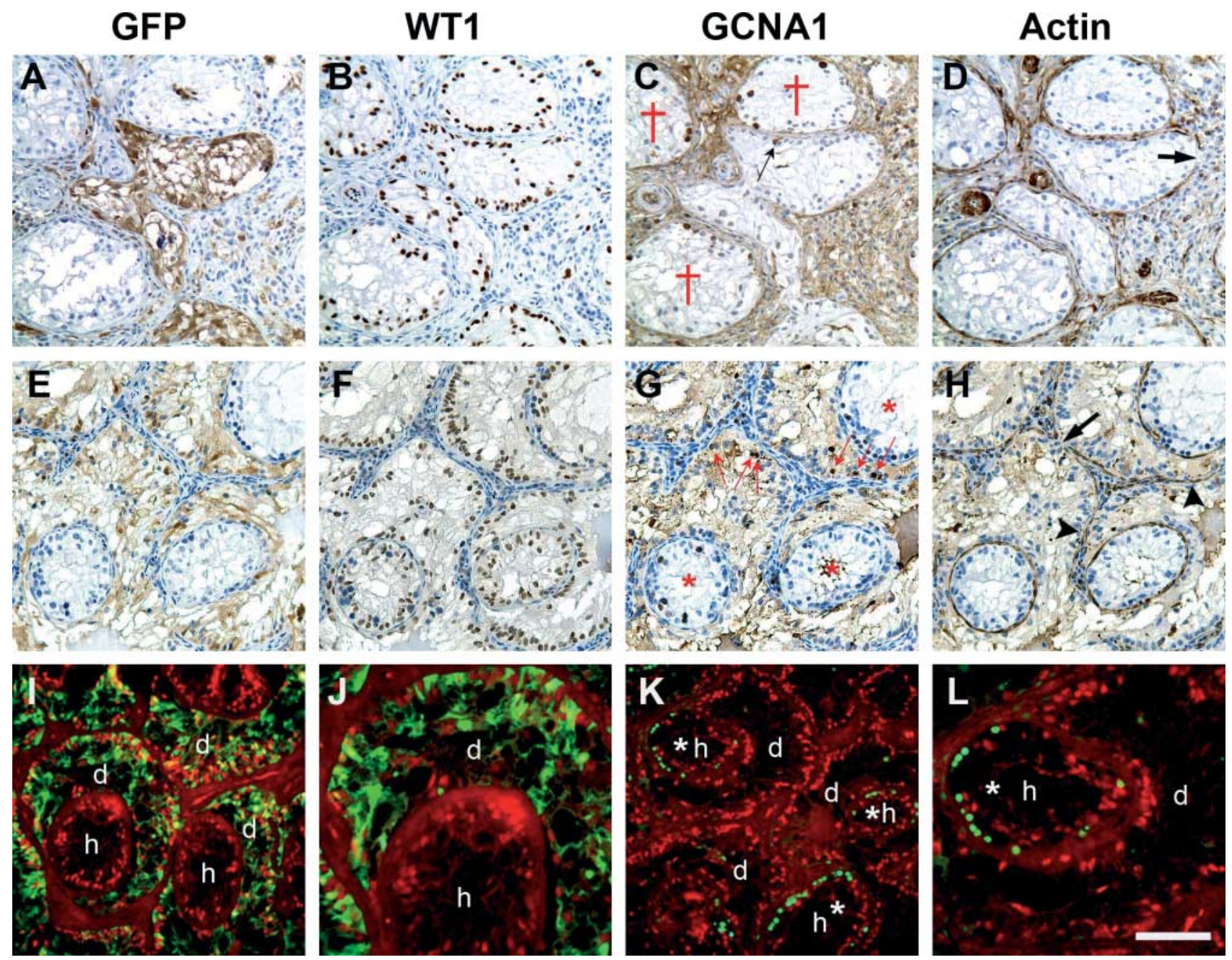

Figure 2 Characterization of tubule-like structures produced by Sertoli cells inadvertently (A-D) or deliberately (E-L) injected into the interstitial space by immunohistochemical staining of donor cells with anti-GFP (A and E, green in I and J), Sertoli cells with anti-WT1 (B, F, red in I-L), germ cells with anti-GCNA (C and G), peritubular myoid cells with anti-smooth muscle $\alpha$-actin ( $D$ and $H$ ), and proliferating cells with anti-PCNA (green in $\mathrm{K}$ and $\mathrm{L}$ ). Bar $=100 \mu \mathrm{m}$ in all panels except $\mathrm{J}$ and $\mathrm{L}$, in which it represents $50 \mu \mathrm{m}$. Arrowheads indicate peritubular myoid layer between de novo formed tubules, and thick arrows indicate gaps in that layer. Thin arrows indicate spermatogonia in de novo formed tubules. Daggers ( $t$ ) indicate host tubules containing type A spermatogonia as the only germ cells and asterisks $(*)$ indicate those tubules containing differentiating cells as well. In $(\mathrm{I}-\mathrm{L})$, ' $\mathrm{d}$ ' indicates newly formed tubule-like structures derived from donor Sertoli cells and ' $\mathrm{h}$ ' indicates tubules consisting of host cells.

since they were GFP negative, represent induced recovery of host spermatogenesis. Many of these differentiated germ cells were well stained with GCNA1, which preferentially marks spermatogonia and early spermatocytes in rat testes. Additional round cells closer to the lumen, which were unstained or palely stained with GCNA1, were likely early spermatids. In other tubules (not shown), endogenous spermatogenesis had progressed to the late spermatid stage. This was unexpected since 6 Gy of irradiation of $\mathrm{LBNF}_{1}$ rats reduces spermatogonial counts to one spermatogonium per seven tubule cross sections, and the percentage of tubules with any differentiated germ cells beyond the $A$ spermatogonial stage to less than $0.1 \%$ (Shuttlesworth et al. 2000).
In addition, when donor Sertoli cells formed colonies in the lumen of tubules (Fig. 4D and E), as evidenced by GFP and vimentin staining, differentiation of endogenous germ cells was occasionally stimulated in the same tubule. In addition to numerous basally located type B spermatogonia that strongly stained with GCNA1 (thin arrows), late pachytene spermatocytes (thick arrows) and round spermatids (arrowheads) that stained weakly for GCNA1 were observed (Fig. 4F).

The formation of individual de novotubules from donor Sertoli cells by accidental injection of cells into the interstitial space during rete testis injection (Fig. 4G and $\mathrm{H})$ also induced differentiation of endogenous spermatogonia (GFP negative) in the adjacent tubules of the recipient testis. These spermatogonia (arrows) and the 


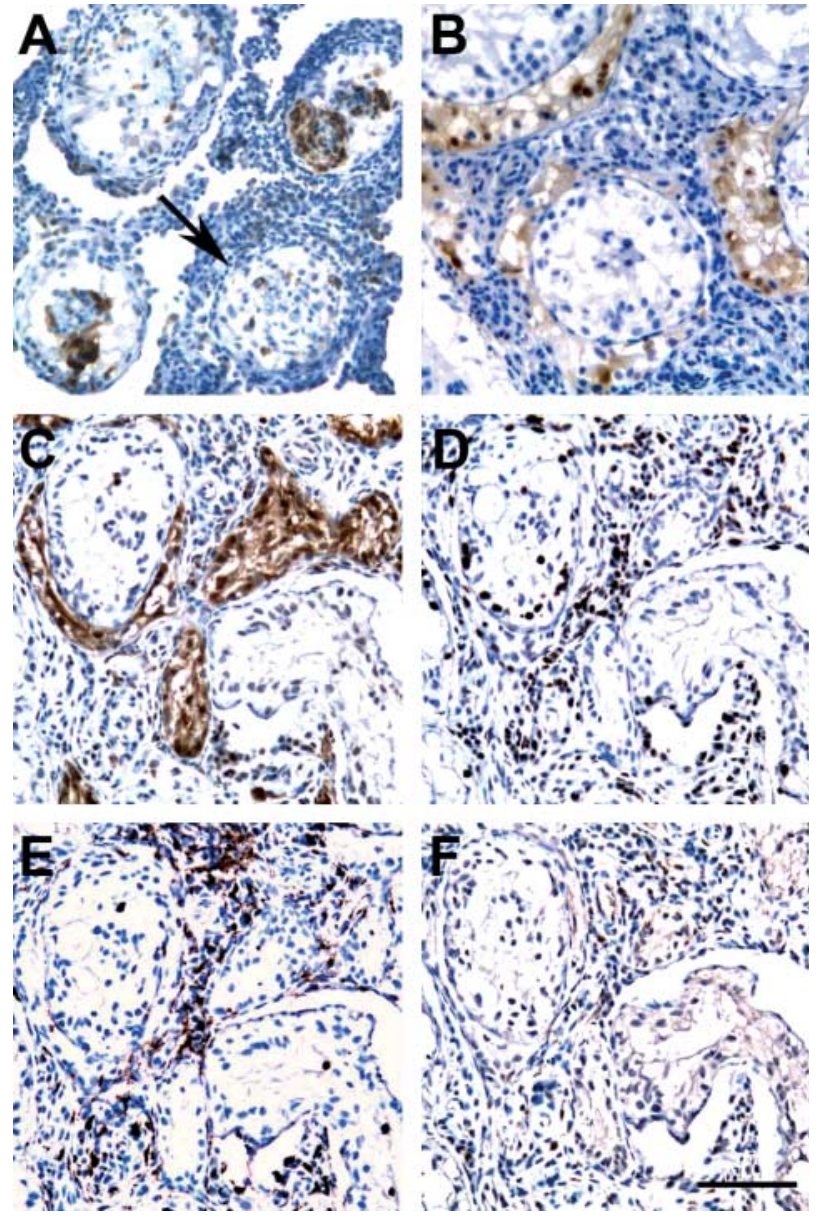

Figure 3 Excessive development of interstitial cells around tubules containing donor Sertoli cells (A) and de novo formed tubules containing donor Sertoli cells (B-F). (C-F) Serial sections. (A-C) AntiGFP staining for donor cells. (D) Anti-PCNA staining for proliferating cells. (E) Anti-ED1 for circulating macrophages. (F) Anti-ED2 for tissueresident macrophages. Arrow indicates degenerating tubule. Bar $=100 \mu \mathrm{m}$.

early primary spermatocytes (dark brown) stained positively with anti-GCNA1 (Fig. 4I). Even when most of the injected cells inadvertently went into the interstitial space and formed, in some regions, a network of de novo tubule-like structures in the interstitial space (Fig. 4J and $\mathrm{K})$, differentiation of endogenous spermatogonia in some of the embedded preexisting tubules was stimulated. This was indicated by the large number of GCNA1-positive cells (arrows) at or near the basement membrane (Fig. 4L).

\section{Stimulation of endogenous spermatogenesis: quantitative analysis}

We then quantified the percentages of tubules showing colonization by Sertoli cells and those showing differentiation of endogenous spermatogenic cells after different types of injection (Table 1). Although a range of backcross generations of donors (three to seven generations) and times between irradiation and transplantation (7-21 weeks) were used, these variations had no effect on the percentages of tubules showing colonization by Sertoli cells or the stimulation of endogenous spermatogenesis. There were, however, apparent increases in the colonization and stimulation with increasing donor age (range, 5-21 days). The most likely cause of this was the larger numbers of viable cells obtained and injected when older rats were used, since when the values were normalized to the average numbers of cells injected in each treatment group, there was no longer any significant dependence of Sertoli cell colonization (Fig. 5A) or endogenous differentiation (not shown) on donor age. Surprisingly, there was no difference between the colonizing efficiency of Sertoli cells from 5- to 6-day-old and 19- to 21-day-old rats and, in some recipients, normally postmitotic Sertoli cells from 20- to 21-day-old rats colonized more than 40 and $20 \%$ of tubules (before normalization) respectively.

When we performed a sham transplantation and injected the testes with either medium or nothing at all, there was little or no differentiation of endogenous germ cells (Table 1), as was the case with unmanipulated irradiated rats (Shuttlesworth et al. 2000). However, when the donor cells were injected through the rete testes and Sertoli cell colonization occurred either within the tubules (intratubular), both within and outside the tubules (intratubular plus interstitial), or outside of the tubules forming de novo tubules (inadvertent interstitial), there were significant increases in endogenous spermatogonial differentiation in $3-4 \%$ of the tubules (Table 1 ). The significant correlation between the percentages of tubules showing differentiation of endogenous germ stem cells and the percentages of tubules showing Sertoli cell colonies in the 45 rat testes receiving only intratubular injection of donor cells (Fig. 5B) provided support for the hypothesis that the Sertoli cell colonization was the likely cause of the stimulation of endogenous spermatogonial differentiation.

When the donor cells were deliberately injected into the interstitium, a significantly higher percentage (14\%) of the tubules showed differentiation of endogenous spermatogonia than when injections were performed through the rete testis. This was perhaps, in part, due to the larger volume of donor cells injected into the interstitial compartment (about 400-800 $\mu \mathrm{l}$ ) than that injected during unsuccessful attempted injections through the rete testis (which was limited to $400 \mu \mathrm{l}$ ). However, significant differences were also observed in the normalized tubule differentiation index, which was obtained by correcting for the numbers of cells injected in the different transplantations, indicating that the greater efficiency of interstitial injection at stimulating endogenous spermatogenesis was not merely a result of the different numbers of cells injected (Table 2). 


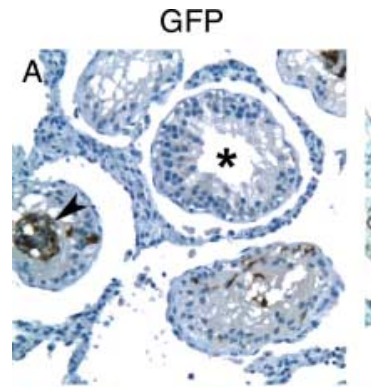

WT1 or vimentin
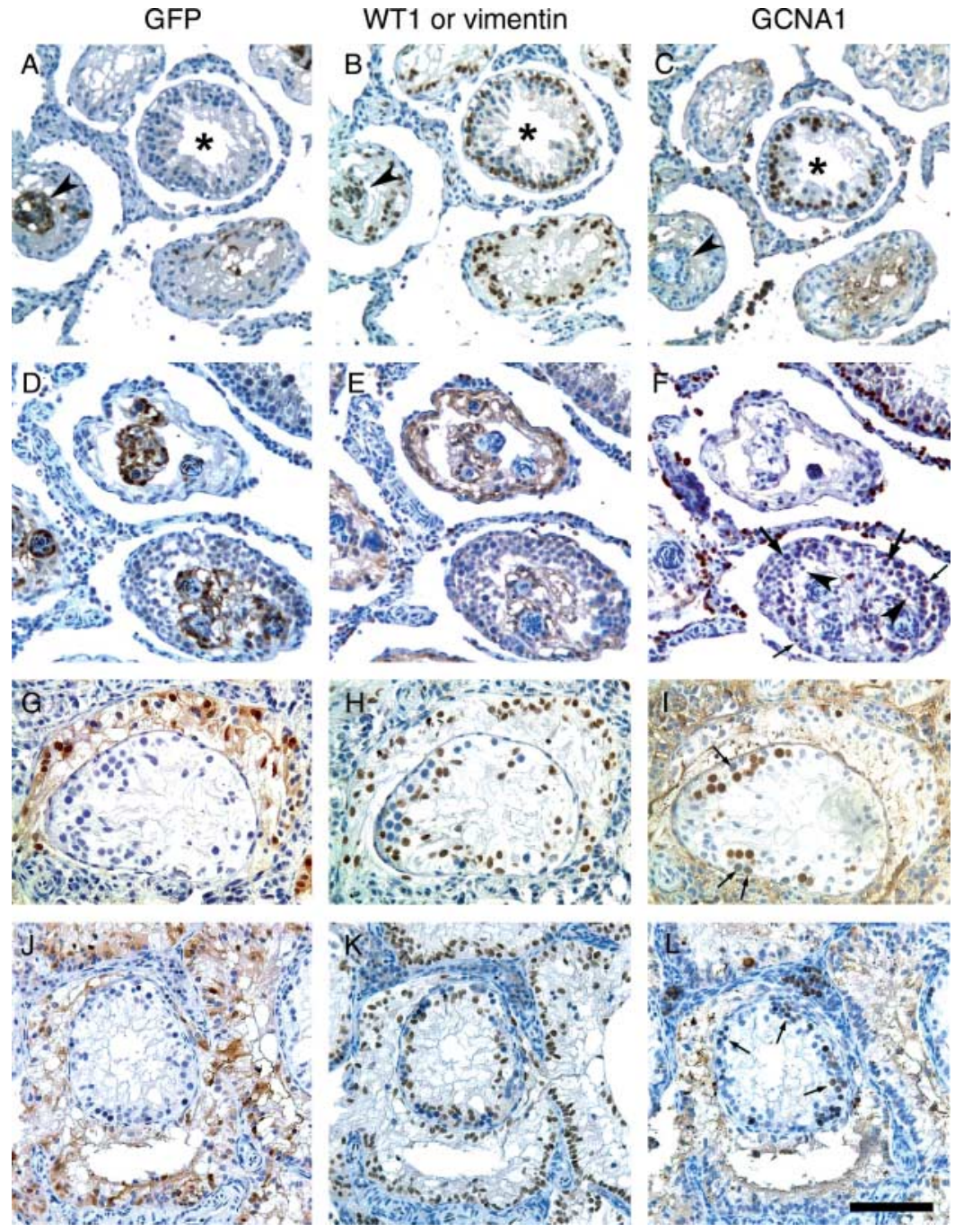

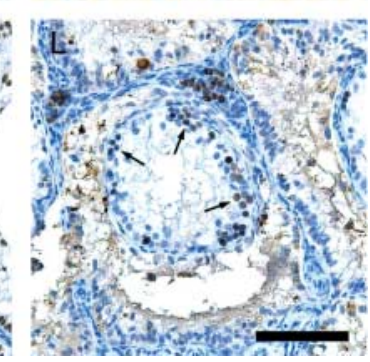

Figure 4 Stimulation of endogenous spermatogonial differentiation by colonization with transplanted Sertoli cells. Serial sections are stained with anti-GFP (A, D, G and J) to show donor cells, with anti-WT1 (B, $\mathrm{H}$ and $\mathrm{K}$ ) or vimentin (E) to show Sertoli cells, and with anti-GCNA1 to show germ cells (C, F, I and L). (A-C) Colonization of tubules by donor Sertoli cells (arrowheads) stimulates differentiation in adjacent tubules $(*)$. (D-F) Colonization of tubules by donor Sertoli cells in the lumen occasionally stimulates endogenous differentiation in the same tubule. (G-I) De novo tubule-like structures formed by accidental injection of donor Sertoli cells (from 21-day-old rat) into the interstitium stimulates differentiation of endogenous spermatogonia in host tubule. (G-I) De novo tubule-like structures formed by deliberate injection of donor Sertoli cells (from 12-day-old rat) into the interstitium stimulates differentiation of endogenous spermatogonia in adjacent host tubule. Bar $=100 \mu \mathrm{m}$ in $(\mathrm{A}-\mathrm{F}),(\mathrm{J}-\mathrm{L})$ and $80 \mu \mathrm{m}$ in $(\mathrm{G}-\mathrm{I})$. In (F, I, and $\mathrm{L})$, arrowheads point to round spermatids, thin arrows point to spermatogonia, and thick arrows point to spermatocytes.

\section{Discussion}

In this study, we have further described the colonization of irradiated rat testis by Sertoli cells collected from testis tubules of immature rats and transplanted along with spermatogonia (Zhang et al. 2007). The donor Sertoli cells had the ability both to colonize the interior of tubules, either as structures on newly formed basement membrane in the center of the tubules or partially or completely replacing the endogenous Sertoli cells along the basement membrane, and to form new tubule-like structures in the interstitium.

The structures we observed in the lumen of the tubules were quite different from those that developed following transplantation of perinatal rat seminiferous tubule cells into recipient rat testes (Jiang \& Short 1995), which were intralumenal, truncated segments of seminiferous epithelium containing basement membrane, Sertoli cells, and complete spermatogenesis. Those structures also showed interdigitation with the host's seminiferous epithelium, with the basement membranes sometimes dividing the tubule into multiple sections (Jiang \& Short 1998). Although it was not possible to prove unequivocally that the luminal spermatogenesis was donor derived, such structures were only observed after transplantation.

Our observation of the ability of Sertoli cells from young rats to form de novo tubule-like structures in the testicular interstitium is consistent with the previous reports of the development of testicular tubular structures when immature rodent, porcine, or bovine testicular cells, as pellets or following re-aggregation in culture, were implanted under the back skin or testis tunica albuginea of nude mice (Gassei et al. 2006, Honaramooz et al. 2007, Kita et al. 2007, Zhang et al. 2008).

In the present study, despite the decreasing percentage of Sertoli cells in testicular tubule cell suspensions and their lower proliferative potential with increasing age, there was no significant decline in the ability of Sertoli 

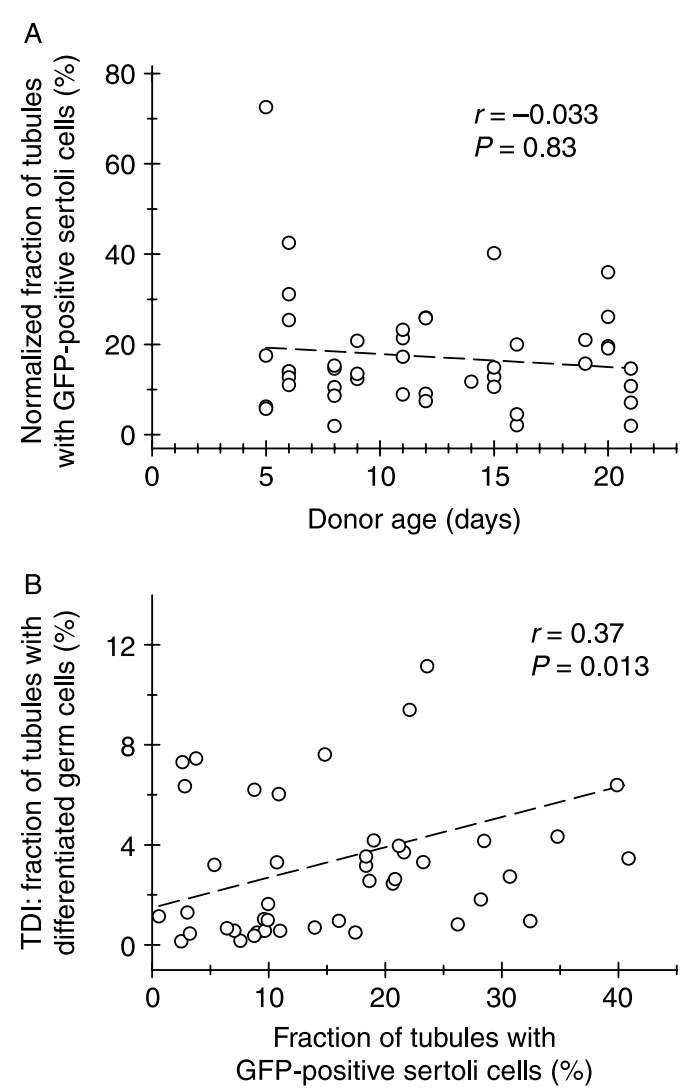

Figure 5 Relationships involving the percentages of tubules showing colonization by donor Sertoli cells after injection of tubular cells from immature GFP-transgenic rats through the rete testis of the recipients. (A) Lack of correlation of the normalized (for the number of cells injected) percentages of tubules showing donor Sertoli cell colonization with donor age. (B) Significant correlation of the percentage of tubules showing differentiation of endogenous germ cells with the percentage of tubules showing donor Sertoli cell colonization. In this panel, data were not normalized for numbers of cells injected. cells from donor rats up to 21 days of age to colonize the tubules (Fig. 5A). Large areas of epithelium in existing or de novo formed tubules contained donor Sertoli cells at a greater density than that observed with the endogenous cells (Fig. 1N, Fig. 2F and Fig. 4K). Although we did not deliberately eliminate Sertoli cells as done in other studies to make room for proliferation of donor cells (Shinohara et al. 2003), the high density of donor Sertoli cells that colonized in the host's tubules indicated that the seminiferous epithelium had space to allow a greater number of Sertoli cells along the basement membrane.

Understanding the relationship between the different patterns of Sertoli cell colonization and spermatogonial differentiation may help elucidate the cellular damage responsible for the block in spermatogonial differentiation in irradiated rats and its reversibility. It is notable that when donor Sertoli cells colonized in a physiological location, along the basement membrane of recipient tubules, they did not support or stimulate spermatogonial differentiation, whereas when they colonized ectopic locations, they stimulated endogenous spermatogonial differentiation in neighboring tubules.

It is important to consider that the spermatogonial stem cell niche is composed of Sertoli cells and basement extracellular matrix, and possibly peritubular myoid and interstitial cellular components around the seminiferous tubules (Shetty \& Meistrich 2007, Yoshida et al. 2007). The observation of isolated donor (Fig. $1 \mathrm{G}$ and $\mathrm{K}$ ) and host (Fig. 1N) spermatogonial cells associated with Sertoli cells that colonized along the tubular basement membranes and the observation of donor spermatogonia with the Sertoli cells forming the de novo tubules along basement membranes in the interstitial compartment (Fig. 2C and G) demonstrates that the donor Sertoli cells formed new niches for spermatogonial stem cells. In the

Table 1 Stimulation of differentiation of endogenous spermatogenic cells. Dependence on mode of injection ${ }^{\mathrm{a}}$.

\begin{tabular}{|c|c|c|c|c|c|c|c|c|}
\hline Type of injection & $\begin{array}{l}\text { Number } \\
\text { of testes }\end{array}$ & $\begin{array}{c}\text { Injection } \\
\text { time, weeks } \\
\text { post- } \\
\text { irradiation } \\
\text { of recipient }\end{array}$ & $\begin{array}{l}\text { Donor } \\
\text { age } \\
\text { (days) }\end{array}$ & $\begin{array}{l}\text { Number of } \\
\text { viable cells } \\
\text { injected }\end{array}$ & $\begin{array}{c}\text { Backcross } \\
\text { generation } \\
\text { of donor }\end{array}$ & $\begin{array}{l}\text { Fraction of } \\
\text { host tubules } \\
\text { containing } \\
\text { GFP-positive } \\
\text { cells }^{\text {b }(\%)}\end{array}$ & $\begin{array}{c}\text { Tubule } \\
\text { differentiation } \\
\text { index }(\mathrm{TDI})^{\mathrm{C}}\end{array}$ & $\begin{array}{c}\text { Normalized } \\
\text { tubule } \\
\text { differentiation } \\
\text { index }(\mathrm{TDI})^{\mathrm{c}, \mathrm{d}}\end{array}$ \\
\hline Control $^{\mathrm{e}}$ & 9 & $9.7 \pm$ & - & - & - & 0 & $0.5 \pm 0.2^{\S}$ & $0.5 \pm 0.2^{\S}$ \\
\hline Intratubular & 45 & $10.7 \pm 0.4$ & $12 \pm 1$ & $20( \pm 1) \times 10^{6}$ & $5.1 \pm 0.2$ & $18.7 \pm 1.8$ & $3.4 \pm 0.8^{\dagger, \S}$ & $3.9 \pm 0.8^{\dagger, \neq}$ \\
\hline Intratubular plus interstitial ${ }^{f}$ & 6 & $10.1 \pm 1.0$ & $13 \pm 3$ & $15( \pm 5) \times 10^{6}$ & $5.7 \pm 0.8$ & $6.2 \pm 0.3$ & $3.7 \pm 1.6^{* \prime} \neq$ & $5.1 \pm 1.8^{+}$ \\
\hline Interstitial (inadvertent) ${ }^{\mathrm{g}}$ & 10 & $9.9 \pm 0.6$ & $11 \pm 2$ & $18( \pm 3) \times 10^{6}$ & $5.9 \pm 0.2$ & $0.1 \pm 0.1$ & $3.0 \pm 1.3^{\ddagger}$ & $2.9 \pm 0.4^{* \prime} \neq$ \\
\hline Interstitial (deliberate) & 4 & $9.9 \pm 0.7$ & $13 \pm 1$ & $32( \pm 4) \times 10^{6}$ & $7.0 \pm 0.0$ & $\overline{0}$ & $14.1 \pm 2.9^{+}$ & $9.0 \pm 1.7^{+}$ \\
\hline
\end{tabular}

Significance of differences in TDI: significantly different from control at $* P<0.05$ or $^{\dagger} P<0.005$. Significantly different from interstitial injection at ${ }^{\ddagger} P<0.05$ or ${ }^{\S} P<0.005$.

${ }^{a}$ Values given as mean \pm S.E.M. ${ }^{b}$ We previously showed that $83 \%$ of GFP-positive tubules contain donor Sertoli cells, with the remaining $17 \%$ containing donor spermatogonia; none of the differentiating germ cells were GFP positive. ${ }^{\mathrm{C}}$ The tubule differentiation index is the percentage of tubules showing differentiation in at least the B spermatogonial stage. All of the germ cells in tubules showing differentiation were GFP negative. ${ }^{\mathrm{d}}$ Tubule differentiation indices were normalized by dividing by the number of viable cells injected and multiplying by the average number of cells injected in all transplantations $\left(20 \times 10^{6}\right)$. ${ }^{e}$ Controls included testes injected with medium alone $(250-350 \mu l$, $n=6)$ and rats that were not injected $(n=3) .{ }^{\mathrm{f}}$ Testes that were injected into the rete testis ( $>1 \%$ of tubules contain GFP-positive cells) but some of the solution leaked into the interstitial space. ${ }^{g}$ Testes that were injected into the rete testis but most of the suspension went into the interstitial space $(<1 \%$ of the tubules contained GFP-positive cells). 
Table 2 Stage of development of differentiated endogenous spermatogenic colonies.

\begin{tabular}{lccc}
\hline & \multicolumn{2}{c}{ Tubular differentiation index (TDI) to indicated stage or beyond (\%) } \\
\cline { 2 - 4 } Type of injection $^{\mathrm{a}}$ & B-spermatogonia & Spermatocytes & Spermatid \\
\hline Control & $0.5 \pm 0.2^{\S}$ & $0.06 \pm 0.03^{\S}$ & 0 \\
Intratubular & $3.4 \pm 0.8^{+, \S}$ & $1.4 \pm 0.4^{+, \S}$ & $0.6 \pm 0.3^{*}$ \\
Intratubular plus interstitial & $3.7 \pm 1.6^{*, \neq}$ & $1.1 \pm 0.7^{\ddagger}$ & 0 \\
Interstitial (inadvertent) & $3.0 \pm 1.3^{\ddagger}$ & $0.8 \pm 0.3^{\S}$ & 0 \\
Interstitial (deliberate) & $14.1 \pm 2.9^{+}$ & $7.1 \pm 1.7^{+}$ & 0 \\
\hline
\end{tabular}

Values given as mean \pm S.E.M. Significance of differences in TDI: significantly different from control at $* P<0.05$ or ${ }^{\dagger} P<0.005$. Significantly different from interstitial injection at ${ }^{\ddagger} P<0.05$ or ${ }^{\S} P<0.005$.

${ }^{\text {a }}$ Same definitions of injection types and numbers of testes as in Table 1.

irradiated testes, these spermatogonia in such niches did not show differentiation within 3 months after transplantation. Furthermore, although donor Sertoli cells often colonized at a high density, the number of spermatogonia in the tubules still appeared to be quite low. Similar to our present report, we found earlier that bovine Sertoli cells transplanted into nude mice either within the testis tubules or in the interstitium of the testis did not support bovine spermatogonial differentiation (Zhang et al. 2008). Although others have observed that niches formed in mouse seminiferous tubules by transplantation of mouse Sertoli cells (Shinohara et al. 2003) and in de novo formed tubules from testicular cell suspensions from both mice and rats transplanted subcutaneously (Kita et al. 2007) did support spermatogenesis, this was only observed in a few tubules.

While these results may indicate that in our case the donor Sertoli cells and the niche that they formed were not functional, the failure of spermatogonia associated with the donor Sertoli cells to differentiate in this environment is consistent with our previous observations that the somatic environment does not support the differentiation of either endogenous (Shuttlesworth et al. 2000) or donor spermatogonia (Zhang et al. 2007) in the host seminiferous tubules of irradiated rats unless testosterone is suppressed. That the normal donor Sertoli cells injected into irradiated rat testes were not able to either establish functional spermatogonial stem cell niches or support spermatogonial differentiation directly in this environment suggests that the somatic cellderived factors that inhibit spermatogonial differentiation in the irradiated rat testis may have been derived from other cells, besides Sertoli cells. However, to conclusively prove this it would be necessary to demonstrate that the transplanted Sertoli cells are indeed functional in a more permissive environment.

By contrast, colonization of irradiated rat testis tubules with Sertoli cells from immature testis tubules at ectopic positions, either in the center of the seminiferous tubules or in de novo formed tubules in the interstitium, stimulated differentiation of some endogenous spermatogonial stem cells. We ruled out that the transplantation surgery alone accounted for differentiation of any arrested spermatogonia in the irradiated testes by performing sham injections. We propose that it is the colonization by transplanted Sertoli cells, either in the intratubular environment or in de novo formed tubules, which indirectly provides the stimulus for inducing the differentiation. This proposal is supported by the positive correlation between the fraction of tubules with GFPpositive Sertoli cells and the fraction of tubules with differentiated germ cells after intratubular injection, and by the observations that differentiation of endogenous spermatogonia always occurs in tubules adjacent to (Fig. 4C) or occasionally within (Fig. 4F) those tubules containing donor Sertoli cells in a non-physiological location, or in host tubules adjacent to de novo tubules containing donor Sertoli cells (Fig. $4 \mathrm{I}$ and $\mathrm{L}$ ) that were formed outside of the host tubules.

One clue to the mechanism of this stimulation is that, in the regions surrounding both the tubules containing Sertoli cell colonies (Fig. 1I, 3A and 4D) and the de novo formed tubules (Fig. 4C), there was a moderate increase in interstitial cell numbers. The cells were suggestive of a chronic inflammatory reaction, and not an acute inflammation, which would be characterized by densely packed small lymphocytes, although we cannot rule out that earlier there was an acute inflammation in these regions of the testis that resolved prior to the 13 -week time point, as no tissue was harvested at earlier time points. Macrophages, particularly the circulating ED1-positive ones as opposed to resident ED2-positive cells, were enriched in these interstitial regions (Fig. $3 \mathrm{E}$ and $\mathrm{F}$ ). Whereas resident macrophages are essential for the normal proliferation and maturation of Leydig cells, peripheral inflammatory macrophages produce cytokines (such as IL1, IL6, and TNF) and reactive oxygen species that inhibit Leydig cell steroidogenesis (Hales 2002), reducing the local tissue levels of testosterone, which allows spermatogonial differentiation to proceed (Shetty et al. 2000). It is also possible that an increase in non-Leydig-cell interstitial cellularity and the de novo formed tubules in the interstitium segregate the testosterone-producing Leydig cells from the host seminiferous tubules, which also reduces local 
testosterone levels. Furthermore, inflammatory reactions, which often involve macrophage recruitment, result in reductions in the interstitial edema (Hedger et al. 2005) that appears to be a major factor blocking spermatogonial differentiation in irradiated rat testes (Porter et al. 2006). Thus, the mild chronic inflammatory reaction resulting from transplantation and colonization of donor Sertoli cells in abnormal locations could be responsible for the increase in spermatogonial differentiation, but the exact mechanism cannot yet be determined.

This study has shown that Sertoli cells from immature rats can form colonies and epithelial structures within the tubules and in the interstitium of irradiated rats and thereby stimulate recovery of spermatogenesis from surviving endogenous stem cells that were otherwise blocked from differentiating by the action of testosterone in these damaged testes. Since spermatogonia within the stem cell niches created by relatively normal epithelia formed by these Sertoli cells (Fig. 1J and N) did not undergo differentiation, it is unlikely that these Sertoli cells produce a juxtacrine or paracrine factor that directly stimulates the spermatogonia. It is more likely that the Sertoli cells in abnormal locations secreted factors that acted indirectly on the interstitium to produce a mild chronic inflammation, possibly involving macrophages, which reduced local testosterone or interstitial fluid levels and then allowed spermatogonial differentiation to proceed.

It is not clear whether or not there is a potentially reversible block of spermatogonial differentiation in the human testis after cytotoxic exposure. The occasional observation of isolated spermatogonia in testicular biopsies at relatively long times after chemotherapy treatment (Kreuser et al. 1989) and the spontaneous recovery of spermatogenesis in some men after several years of radiation or chemotherapy-induced azoospermia (Hahn et al. 1982, Pryzant et al. 1993, Gerdprasert et al. 2002) indicate that there may indeed be a reversible block to differentiation in humans. Sertoli cell transplantation may still be useful for treatment of infertility induced by radiation or antineoplastic drugs to reverse this block and provide additional niches for co-transplanted stem cells. Further study of the ectopic colonization by Sertoli cells may provide information with both basic and clinical applications.

\section{Materials and Methods}

\section{Animals}

$\mathrm{LBNF}_{1}$ rats $\left(\mathrm{F}_{1}\right.$ hybrids of Lewis and Brown-Norway; Harlan Sprague-Dawley, Indianapolis, IN, USA) were used as recipients. $\mathrm{LBNF}_{1}$ rats were chosen because they are an $F_{1}$ hybrid between two inbred strains, and therefore have hybrid vigor and can accept transplants from either parental strain, and are sensitive to the hormone-mediated block in spermatogonial differentiation induced by radiation and other cytotoxic agents (Parchuri et al. 1993, Gerdprasert et al. 2002, Meistrich \& Shetty 2003). However, LBNF $_{1}$ is not unique as other inbred and outbred strains of rats, including Lewis, PVG, certain substrains of Wistar, Fischer 344, and Sprague-Dawley also have a high sensitivity to radiation and anticancer agents (Delic et al. 1986a, 1986b, Ward et al. 1990, Gerdprasert et al. 2002), show this block in spermatogonial differentiation after exposure to toxicants (Allard et al. 1996), and restore spermatogenic recovery from toxicant exposure upon suppression of hormones (Delic et al. 1986a, 1986b, Gerdprasert et al. 2002, Udagawa et al. 2006).

Donors were transgenic rats expressing GFP under the control of a CMV enhancer and ubiquitin-C promoter (Lois et al. 2002). The GFP-transgenic rats, originally on a Sprague-Dawley genetic background, were backcrossed to the inbred Lewis strain (Harlan Sprague-Dawley). The generation of the same allele at the major histocompatibility complex locus RT1 was monitored using PCR and agarose gel analysis of the closely linked microsatellite locus D20Rat46 (Rat Genome Database, http://rgd.mcw.edu/). At the second generation of the backcross, all of the rats used for further breeding had the haplotype of the Lewis strain and had lost the Sprague-Dawley allele (data not shown). Therefore, some of the backcrossed GFP-Lewis males from the third, fourth, and fifth generations were used as donors for testicular cell transplantation to $\mathrm{LBNF}_{1}$ rats. GFP-positive females from the fifth and sixth Lewis-backcross generation were mated with Brown-Norway male rats (Harlan SpragueDawley) to obtain $\mathrm{LBNF}_{1}$ rats expressing GFP. All animals were caged in a controlled environment at M D Anderson Cancer Center (12h light:12h darkness) with unlimited access to food and water. All experiments were approved by the Institutional Animal Care and Use Committee of the University of Texas M D Anderson Cancer Center.

\section{Preparation of recipients}

Male $\mathrm{LBNF}_{1}$ rats (about 8 weeks old) were anesthetized with an i.m. injection of a ketamine $(0.72 \mathrm{mg} / \mathrm{kg})$ and acepromazine $(0.022 \mathrm{mg} / \mathrm{kg})$ mixture and irradiated with a ${ }^{60} \mathrm{Co} \gamma$-ray unit (Eldorado 8; Atomic Energy Canada Ltd, Ottawa, Ont., Canada) as described previously (Shetty et al. 2000). Rats were placed on their backs, and $5 \mathrm{~mm}$ of tissue-equivalent bolus material (Superflab, Mick Radio-Nuclear Instruments Inc., Bronx, NY, USA) was placed over the scrotum to provide a buildup layer. The irradiation field extended anteriorly about $6 \mathrm{~cm}$ above the base of the scrotum. A single dose of 6 Gy was given at a dose rate of about $0.9 \mathrm{~Gy} / \mathrm{min}$. The rats were used as recipients for germ cell transplantation between 7 and 21 weeks after irradiation.

\section{Preparation of donor cells}

Immature rats (5-21 days old) were used as testicular cell donors. To harvest single cells from the tubules, we removed the tunica and sequentially digested the tissue with enzymes at $35{ }^{\circ} \mathrm{C}$ in a shaking water bath as described in detail recently (Zhang et al. 2006). Tubules were prepared by two digestions, 
first with collagenase and then with collagenase and hyaluronidase in DMEM/F12 medium containing DNase I and fetal bovine serum for 20-30 min each. Tubules were then digested with trypsin in Dulbecco's PBS (Gibco) containing 1 mM EGTA and DNase I for 10-15 min. The final pellets were resuspended in DMEM/F12 containing 10\% fetal bovine serum.

Trypan blue (Gibco) was added to a concentration of $0.02 \%$. After the cell concentration and viability (Trypan blueexcluding cells) were determined, the cell suspension was kept on ice until transplantation.

\section{Transplantation}

Testes of anesthetized recipient rats were exteriorized through an abdominal incision, a 30-G dental needle (Terumo Corporation, Tokyo, Japan) was inserted into the efferent ducts, and donor cells were injected using a $1 \mathrm{ml}$ syringe. Between 100 and $400 \mu$ of the cell suspension was injected into each recipient testis. The total number of viable cells injected into recipient testis was calculated based on cell viability, concentration, and volume injected and is summarized in Table 1. Transplantation was monitored with the Trypan blue to indicate whether the cell suspension was injected into the seminiferous tubules or out of the seminiferous tubules.

A total of 61 irradiated rat testes were injected with donor cells through the efferent ducts; among them, 45 testes were successfully injected with donor cells exclusively into the seminiferous tubules, 6 testes received donor cells to areas both inside and outside of the seminiferous tubules, and 10 testes received donor cells only in the interstitial compartment. In addition, four testes were deliberately injected with donor cells in the interstitium by inserting the needle into one or two random points of the testis and moving the needle during injection. An additional three irradiated testes were not injected at all and six were injected with only medium (the same medium as used for the cell suspension, with Trypan blue) into the seminiferous tubules.

\section{Microscopic and immunohistochemical assessment}

Recipients were killed 13 weeks after transplantation. After removal of the tunica albuginea, the testes were fixed in $4 \%$ paraformaldehyde (Sigma-Aldrich) solution overnight at $4{ }^{\circ} \mathrm{C}$ and subjected to routine histological processing, embedding, and sectioning at 4-5 $\mu \mathrm{m}$ thickness.

Serial sections were prepared for immunohistochemistry, including antigen retrieval, inhibition of endogenous peroxidase, and blocking as described previously (Zhang et al. 2006). Rabbit polyclonal (1:5000 dilution, Novus Biologicals, Littleton, CO, USA) or mouse monoclonal (1:1000 dilution, or Roche-Applied Science) anti-GFP was used to stain donor Sertoli and germ cells from the transgenic rats. The rat antimouse monoclonal anti-GCNA1 (germ cell nuclear antigen 1) antibody (1:100 dilution, a gift from Dr George Enders) or rabbit anti-mouse polyclonal antibody to DDX4/Mvh (Vasa) (1:3000 dilution, Abcam, Cambridge, UK) was used to stain germ cells. The rabbit polyclonal anti-WT1 (Wilms' tumor gene; 1:1000 dilution, Santa Cruz Biotechnology, Santa Cruz CA, USA) specifically stains nuclei of Sertoli cells at all ages
(Sharpe et al. 2003). The mouse monoclonal anti-smooth muscle $\alpha$-actin (1:2500 dilution, Sigma-Aldrich) specifically stains peritubular myoid cells and smooth muscle in blood vessels. The mouse monoclonal anti-human PCNA antibody (Dako, Glostrup, Denmark) was used to stain proliferating cells. Macrophages were stained with antibodies ED1 (1:100), which recognizes monocytes, dendritic cells, and circulating macrophages, or ED2 (1:50 dilution), which recognizes tissueresident macrophages (AbD Serotec, Raleigh, NC, USA; Gerdprasert et al. 2002).

The primary antibodies were added to tissues and incubated overnight at $4{ }^{\circ} \mathrm{C}$. ABC Elite kits, second antibodies, and 3,3'-diaminobenzidine (all from Vector Laboratories, Burlingame, CA, USA) were used as recommended by the manufacturer. In controls, prepared without the addition of primary antibodies, there was no background staining (not shown). Sections were counterstained with hematoxylin. For fluorescent staining, two primary antibodies were incubated with tissues overnight at $4{ }^{\circ} \mathrm{C}$, followed by incubation with one of the two second fluorescent antibodies (Alexa 488 rabbit anti-mouse or Alexa 546 goat anti-rabbit, 1:300 dilutions, Molecular Probes, Eugene, OR, USA) at room temperature for $1 \mathrm{~h}$. DAPI mounting solution (Vector Laboratories) was used to stain nuclei of all cells. Then results were checked under fluorescence microscopy. Some sections were stained with Masson's trichrome to visualize collagen.

Donor cell-positive tubules were counted in histological sections after anti-GFP staining. The differentiation stages and GFP status of germ cells were determined in each tubule by histological criteria. The data are presented in terms of the tubule differentiation index, which is defined as the percentage of tubules reaching a particular stage of development or beyond. We have previously used the criterion of having at least three germ cells at the B spermatogonial stage or later to consider the tubule as differentiating (Meistrich \& van Beek 1993). However, the identification of the type B spermatogonia in the paraformaldehyde-fixed, immunostained sections is more difficult. We therefore used the criterion containing five or more germ cells, some of which were in chains, near the basement membrane, since they were likely to be type B spermatogonia or very early spermatocytes because undifferentiated type A spermatogonia always appear in sections as single cells or short chains (Russell et al. 1990), to define differentiation to the B spermatogonial stage. The tubular differentiation indices using differentiation to the spermatocyte stage or beyond and to the spermatid stage were also calculated, using the criterion of three or more germ cells that had reached that stage or beyond. All values were based on counting all tubules taken from three sections per testis, at least $100 \mu \mathrm{m}$ apart from each other.

\section{Statistical analysis}

Non-parametric statistics were used because the results of injections (percentages of tubules with donor cell colonization and percentages of tubules showing recovery of endogenous spermatogenesis) were not normally distributed due to the 
variability of the overall procedure. The tubule differentiation indices after the various modes of injection were first compared with the Kruskal-Wallis test and, since there were significant differences among the groups $(P<0.001)$, the groups were then compared pairwise using the Mann-Whitney U-test.

Analysis of correlations ( $P$ values and correlation coefficients) between the percentages of tubules with donor cell colonization or recovery of endogenous spermatogenesis and other factors that might affect the transplant efficiencies was done using a Spearman's rank correlation test. On the scatterplots, a linear regression line is shown for illustrative purposes only.

All statistical analyses were performed using SPSS software.

\section{Declaration of interest}

The authors declare that there is no conflict of interest that could be perceived as prejudicing the impartiality of the research reported.

\section{Funding}

Grant no. ES-08075 from the National Institute of Environmental Health Sciences/NIH (to M L Meistrich), Cancer Center Support grant no. CA-16672, and a Lalor Foundation Fellowship and Australia CSIRO fellowship (to Z Zhang).

\section{Acknowledgements}

We thank Dr G Enders for kindly supplying anti-GCNA1 antibody and Dr R Behringer for supplying the breeder GFP rat. We are also thankful to Jun Ju, Nalini Patel, and Kuriakose Abraham for technical assistance and Walter Pagel for editorial assistance.

\section{References}

Allard EK, Blanchard KT \& Boekelheide K 1996 Exogenous stem cell factor (SCF) compensates for altered endogenous SCF expression in 2,5hexanedione-induced testicular atrophy in rats. Biology of Reproduction 55 185-193.

Delic JI, Bush C \& Peckham MJ 1986a Protection from procarbazineinduced damage of spermatogenesis in the rat by androgen. Cancer Research 46 1909-1914.

Delic JI, Hendry JH, Morris ID \& Shalet SM 1986b Dose and time relationships in the endocrine response of the irradiated adult rat testis. Journal of Andrology 7 32-41.

Gassei K, Schlatt S \& Ehmcke J 2006 De novo morphogenesis of seminiferous tubules from dissociated immature rat testicular cells in xenografts. Journal of Andrology 27 611-618.

Gerdprasert O, O'Bryan MK, Muir JA, Caldwell AM, Schlatt S, de Kretser DM \& Hedger MP 2002 The response of testicular leukocytes to lipopolysaccharide-induced inflammation: further evidence for heterogeneity of the testicular macrophage population. Cell and Tissue Research 308 277-285.

Hahn EW, Feingold SM, Simpson L \& Batata M 1982 Recovery from aspermia induced by low-dose radiation in seminoma patients. Cancer $50337-340$.

Hales DB 2002 Testicular macrophage modulation of Leydig cell steroidogenesis. Journal of Reproductive Immunology 57 3-18.
Hedger M, Klug J, Frohlich S, Muller R \& Meinhardt A 2005 Regulatory cytokine expression and interstitial fluid formation in the normal and inflamed rat testis are under Leydig cell control. Journal of Andrology 26 379-386.

Honaramooz A, Megee SO, Rathi R \& Dobrinski I 2007 Building a testis: formation of functional testis tissue after transplantation of isolated porcine (Sus scrofa) testis cells. Biology of Reproduction 76 43-47.

Jiang FX \& Short RV 1995 Male germ cell transplantation in rats: apparent synchronization of spermatogenesis between host and donor seminiferous epithelia. International Journal of Andrology 18 326-330.

Jiang FX \& Short RV 1998 Different fate of primordial germ cells and gonocytes following transplantation. Acta Pathologica, Microbiologica, et Immunologica Scandinavica 106 58-63.

Kanatsu-Shinohara M, Miki H, Inoue K, Ogonuki N, Toyokuni S, Ogura A \& Shinohara T 2005 Germline niche transplantation restores fertility in infertile mice. Human Reproduction 20 2376-2382.

Kangasniemi M, Huhtaniemi I \& Meistrich ML 1996 Failure of spermatogenesis to recover despite the presence of A spermatogonia in the irradiated LBNF1 rat. Biology of Reproduction 54 1200-1208.

Kita K, Watanabe T, Ohsaka K, Hayashi H, Kubota Y, Nagashima Y, Aoki I, Taniguchi H, Noce T, Inoue K et al. 2007 Production of functional spermatids from mouse germline stem cells in ectopically reconstituted seminiferous tubules. Biology of Reproduction 76 211-217.

Kreuser ED, Kurrle E, Hetzel WD, Heymer B, Porzsolt R, Hautmann R, Gaus W, Schlipf U, Pfeiffer EF \& Heimpel H 1989 Reversible germ cell toxicity after aggressive chemotherapy in patients with testicular cancer: results of a prospective study. Klinische Wochenschrift 67 367-378.

Lois C, Hong EJ, Pease S, Brown EJ \& Baltimore D 2002 Germline transmission and tissue-specific expression of transgenes delivered by lentiviral vectors. Science 295 868-872.

Meistrich ML \& van Beek MEAB 1993 In Methods in Toxicology, pp 106-123. Eds RE Chapin \& J Heindel. New York: Academic Press.

Meistrich ML \& Shetty G 2003 Inhibition of spermatogonial differentiation by testosterone. Journal of Andrology 24 135-148.

Parchuri N, Wilson G \& Meistrich ML 1993 Protection by gonadal steroid hormones against procarbazine-induced damage to spermatogenic function in LBNF1 hybrid rats. Journal of Andrology 14 257-266.

Porter KL, Shetty G \& Meistrich ML 2006 Testicular edema is associated with spermatogonial arrest in irradiated rats. Endocrinology 147 1297-1305.

Pryzant RM, Meistrich ML, Wilson E, Brown B \& McLaughlin P 1993 Longterm reduction in sperm count after chemotherapy with and without radiation therapy for non-Hodgkin's lymphomas. Journal of Clinical Oncology 11 239-247.

Russell LD, Ettlin RA, Hikim APS \& Clegg ED 1990 Histological and Histopathological Evaluation of the Testis, Clearwater, FL: Cache River Press.

Sharpe RM, McKinnell C, Kivlin C \& Fisher JS 2003 Proliferation and functional maturation of Sertoli cells, and their relevance to disorders of testis function in adulthood. Reproduction 125 769-784.

Shetty G \& Meistrich ML 2007 The missing niche for spermatogonial stem cells: do blood vessels point the way? Cell Stem Cell 1 361-363.

Shetty G, Wilson G, Huhtaniemi I, Shuttlesworth GA, Reissmann T \& Meistrich ML 2000 Gonadotropin-releasing hormone analogs stimulate and testosterone inhibits the recovery of spermatogenesis in irradiated rats. Endocrinology 141 1735-1745.

Shinohara T, Orwig KE, Avarbock MR \& Brinster RL 2003 Restoration of spermatogenesis in infertile mice by sertoli cell transplantation. Biology of Reproduction 68 1064-1071.

Shuttlesworth GA, de Rooij DG, Huhtaniemi I, Reissmann T, Russell LD, Shetty G, Wilson G \& Meistrich ML 2000 Enhancement of A spermatogonial proliferation and differentiation in irradiated rats by GnRH antagonist administration. Endocrinology 141 37-49.

Udagawa K, Ogawa T, Watanabe T, Tamura Y, Kita K \& Kubota Y 2006 Testosterone administration promotes regeneration of chemically impaired spermatogenesis in rats. International Journal of Urology $\mathbf{1 3}$ 1103-1108.

Ward JA, Robinson J, Furr BJA, Shalet SM \& Morris ID 1990 Protection of spermatogenesis in rats from the cytotoxic procarbazine by the depot formulation of Zoladex, a gonadotropin-releasing hormone agonist. Cancer Research 50 568-574. 
Yoshida S, Sukeno M \& Nabeshima YI 2007 A vasculature-associated niche for undifferentiated spermatogonia in the mouse testis. Science 317 1722-1726.

Zhang Z, Shao S \& Meistrich ML 2006 Irradiated mouse testes efficiently support spermatogenesis derived from donor germ cells of mice and rats. Journal of Andrology 27 365-375.

Zhang Z, Shao S \& Meistrich M 2007 The radiation-induced block in spermatogonial differentiation is due to damage to the somatic environment, not the germ cells. Journal of Cellular Physiology 211 149-158.
Zhang Z, Hill J, Holland M, Kurihara Y \& Loveland K 2008 Bovine Sertoli cells colonize and form tubules in murine hosts following transplantation and grafting procedures. Journal of Andrology 29 418-430.

Received 16 March 2008

First decision 15 April 2008

Revised manuscript received 10 November 2008

Accepted 25 November 2008 